\title{
Intravascular Volume Modulates the Outcome Predictive Capacity of Clinical Renal Function Biomarkers in Clinically "Euvolemic" Chronic Heart Failure Patients
}

\author{
Wayne L. Miller ${ }^{a}$ Diane E. Grill ${ }^{b}$ Qi Qian ${ }^{c}$ \\ a Department of Cardiovascular Medicine, Mayo Clinic, Rochester, MN, USA; ${ }^{b}$ Biomedical Statistics and Informatics, \\ Mayo Clinic, Rochester, MN, USA; ' Nephrology and Hypertension, Mayo Clinic, Rochester, MN, USA
}

\section{Keywords}

Intravascular volume $\cdot$ Chronic heart failure - Outcomes .

Chronic kidney disease $\cdot$ Clinical biomarkers

\begin{abstract}
Background: Cardiorenal interconnections are complex and may in part be mediated by the extent of intravascular volume expansion. The impact of subclinical volume excess on outcomes in heart failure (HF) patients with chronic kidney disease (CKD) has not been examined previously. Objectives: To assess the impact of volume-kidney interactions on outcomes in clinically "euvolemic" chronic HF patients (NYHA class II) with coexisting CKD. Methods: Plasma volume (PV) was prospectively measured in 110 stable HF patients with different degrees of renal function using a standardized radiolabeled albumin indicator-dilution technique. To examine the interactive roles of volume expansion and biomarkers of CKD, the cohort was dichotomized by median PV and then further stratified by cohort median serum creatinine, eGFR, and BUN, and analyzed for outcomes of HFrelated mortality and 1 st hospitalization. Results: PV was expanded above normal in $76 \%$ of the cohort. Over 1.5 years of follow-up, sCr and BUN above and eGFR below cohort median stratified higher risks for the composite endpoint only in ambulatory HF patients with a severe degree of PV expan-
\end{abstract}

\begin{tabular}{ll}
\hline KARGER & ( 2019 The Author(s) \\
& Published by S. Karger AG, Basel \\
E-Mail karger@karger.com & This article is licensed under the Creative Commons Attribution- \\
www.karger.com/kdd & NonCommercial-NoDerivatives 4.0 International License (CC BY- \\
NC-ND) (http://www.karger.com/Services/OpenAccessLicense). \\
Usage and distribution for commercial purposes as well as any dis- \\
tribution of modified material requires written permission.
\end{tabular}

sion (median PV expansion $\geq+26 \% ; p=0.02$ ). With less expansion $(<+26 \%$ expansion), these biomarkers reflecting worse renal function did not discriminate risk $(p=0.578)$. The percentage of subjects experiencing composite outcome events was, however, comparable for both greater and lesser degrees of PV expansion in HF patients with stable clinical status. Conclusions: In clinically stable chronic HF patients with coexisting CKD, substantial subclinical PV expansion is common even when patients are considered clinically to be euvolemic, and, importantly, the extent of PV expansion impacts outcomes including early HF mortality. Better kidney function appears to mitigate the effects of excess PV expansion, while less volume expansion appears to limit the risk of worse renal function as reflected by clinical biomarkers of renal function. Thus, the extent of volume expansion impacts the capacity of standard clinical biomarkers of CKD to differentiate outcome risk in ambulatory chronic (NYHA class II) HF patients.

(C) 2019 The Author(s)

Published by S. Karger AG, Basel

\section{Introduction}

Persistent clinical volume overload, symptomatic fluid congestion, and renal dysfunction have been hallmarks of poor outcomes and shown to be significant prognostic
Wayne L. Miller, MD, PhD, FACC, FAHA, FHFSA

Department of Cardiovascular Medicine

Mayo Clinic, 200 First Street, SW

Rochester, MN 55905 (USA)

E-Mail miller.wayne@ mayo.edu 
factors in patients with chronic heart failure (HF) [1-3]. However, how volume overload status as reflected in different degrees of subclinical intravascular volume expansion interacts with coexisting chronic kidney disease (CKD) and chronic HF to impact outcomes has not been studied. Also, how the relative severity of volume expansion impacts clinical biomarkers of renal dysfunction to identify risk changes in stable HF remains incompletely examined [4]. Therefore, we sought to assess the volumekidney associations in patients with chronic stable HF and different degrees of renal dysfunction in relation to long-term clinical outcomes of HF mortality and hospitalizations. Our working hypothesis was that severe plasma volume (PV) expansion would be associated with worse renal function and poorer outcomes while lesser degrees of PV expansion would mitigate the risk associated with impaired renal function in ambulatory chronic HF patients.

\section{Methods}

\section{Study Cohort}

Clinically stable patients with known chronic HF, predominately NYHA functional class II, composed the cohort for this analysis of prospectively collected observational data. Nonconsecutive patients evaluated during the period from October 1, 2011, to June 30, 2017, were included in the study. All patients had a quantitative measure of intravascular blood volume (blood volume analysis, BVA) obtained under steady-state clinical conditions. All patients were clinically evaluated as being "euvolemic" and were receiving standard oral HF medical therapy, including $\beta$-blockers, angiotensin converting enzyme inhibitors, or angiotensin receptor blockers, and oral loop diuretics (range of 20-60 $\mathrm{mg}$ /day; furosemide or equivalent) at the time of BVA and laboratory blood testing. Standard blood laboratory test results were available clinically for all patients as well as echocardiographic assessments within 3 months of the BVA. Patient exclusion criteria were (1) age < 18 years; (2) CKD requiring hemodialysis or ultrafiltration; (3) symptomatic coronary artery disease or requiring intravenous or device hemodynamic support; and (4) females who were pregnant or of child-bearing potential.

\section{Intravascular Volume Measurement}

Total blood volume quantitation using the indicator-dilution principle was undertaken in the Mayo Clinical Nuclear Medicine Laboratory using a standardized computer-based clinically available technique to administer low-dose iodinated $\left[{ }^{131} \mathrm{I}\right]$-labeled albumin intravenously. Specifics of the technique and procedure have been reported previously [5-8]. In brief, the radiolabeled albumin is injected, and from the contralateral forearm venous catheter, 6 - $\mathrm{mL}$ blood samples are collected at time 0 (before injection) and $12,18,24,30$, and $36 \mathrm{~min}$ after injection. Plasma radioactivity is measured in duplicate in a semiautomated counter (FDA-approved BVA-100 blood volume analyzer; Daxor Corp., New York, NY, USA). By extrapolating the radioactivity from the samples to
Table 1. Demographic and clinical characteristics of the study cohort $(n=110)$
Age, years

Gender, females/males

Body mass index, $\mathrm{kg} / \mathrm{m}^{2}$

Systolic blood pressure, $\mathrm{mm} \mathrm{Hg}$

Duration of heart failure, months

Left-ventricular ejection fraction, \% Range

Diabetes, \%

Hypertension, \%

Coronary artery disease, $\%$

Atrial fibrillation, \%

Hemoglobin, g/dL

Hematocrit, \%

Serum creatinine, $\mathrm{mg} / \mathrm{dL}$

Median (25th-75th percentile)

eGFR, $\mathrm{mL} / \mathrm{min} / 1.72 \mathrm{~m}^{2}$

Median (25th-75th percentile)

BUN, mg/dL

Median (25th-75th percentile)

Plasma albumin, g/dL

Plasma glucose, $\mathrm{mg} / \mathrm{dL}$

NT-proBNP, pg/mL

\section{$64 \pm 13$}

$31 / 79$

$32 \pm 8$

$112 \pm 18$

$44 \pm 30$

$36 \pm 17$

14-70

40

61

49

49

$12.4 \pm 2.1$

$37 \pm 6$

$1.6 \pm 0.8$

$1.4(1.1-1.8)$

$54 \pm 24$

$51(34-72)$

$36 \pm 23$

29 (19-46)

$3.8 \pm 0.6$

$116 \pm 32$

$3,412(1,175-7,506)$
Means \pm SD or percent in the category unless indicated otherwise. Laboratory data obtained within $24 \mathrm{~h}$ of BVA.

time zero, PV can be measured, and total blood volume calculated from the patient's whole-body hematocrit. This technique is recommended for quantitative assessment of total blood volume by the International Committee for Standardization in Hematology for its precision and reproducibility [9] and also validated against the double-label technique of chromium-tagged red blood cells (RBCs) and $\left[{ }^{125} \mathrm{I}\right]$-labeled albumin [10]. Reference normal volumes are defined by the method of deviation from ideal weight which takes into account differences in body composition. PV values are adjusted for age, sex, weight, and height to calculate normal volumes as derived from $>100,000$ measurements from Metropolitan Life tables. Intravascular volumes are reported as absolute volumes (in liters) and as percent deviations from normal volume as percent volume deficit (-) or excess (+). The cutoff point for severe PV expansion was not established a priori but was based upon the measured median PV of the patient cohort as a whole. The median percent PV expansion for this cohort was $+26 \%$ above normal expected PV. The cohort was thus stratified by PV values above and below the median volume excess. Normal-to-moderate PV expansion included values $<+26 \%$ volume expansion. Normal quantitated PV, total blood volume, and RBC mass are standardly defined as measured volumes within the range from $\geq-10$ to $\leq+10 \%$ of expected normal volumes [7-8].

Patient survival or HF-related mortality was confirmed using the Mayo Clinic, Rochester, electronic medical record system. First HFrelated hospitalization after index BVA was identified by surveillance of electronic medical records and ICD-9-CM codes 425 (cardiomyopathy), 428 (heart failure), and ICD-10-CM 150 (heart fail- 
Table 2. Clinical and demographic characteristics stratified by cohort median (+26\%) plasma volume (PV)

\begin{tabular}{lccc}
\hline Variable & PV expansion & & $\begin{array}{c}\text { Intergroup } \\
\text { comparison, } p\end{array}$ \\
\cline { 2 - 3 } & $\begin{array}{l}<\text { median } \\
(n=55)\end{array}$ & $\begin{array}{c}\geq \text { median } \\
(n=55)\end{array}$ & 0.232 \\
\hline Age, years & $62 \pm 14$ & $65 \pm 12$ & 0.145 \\
Gender, females/males & $20 / 35$ & $10 / 45$ & 1.00 \\
Body mass index, kg/m ${ }^{2}$ & $32 \pm 9$ & $32 \pm 6$ & 0.196 \\
Systolic blood pressure, mm Hg & $111 \pm 15$ & $115 \pm 17$ & 0.117 \\
Left-ventricular ejection fraction, \% & $38 \pm 17$ & $33 \pm 16$ & 0.595 \\
$\quad$ Range & $15-69$ & $14-70$ & 0.457 \\
Diabetes, \% & 37 & 42 & 0.119 \\
Hypertension, \% & 57 & 64 & 0.056 \\
Coronary artery disease, \% & 57 & 42 & 0.078 \\
Atrial fibrillation, \% & 31 & 49 & 0.176 \\
Hemoglobin, g/dL & $12.7 \pm 2.0$ & $12.0 \pm 2.1$ & \\
Serum creatinine, mg/dL & $1.5 \pm 0.6$ & $1.7 \pm 0.9$ & 0.279 \\
$\quad$ Median $(25$ th-75th percentile) & $1.4(1.1-1.7)$ & $1.5(1.1-2.2)$ & \\
eGFR, mL/min/1.72 m ${ }^{2}$ & $51 \pm 25$ & $56 \pm 23$ & 0.162 \\
$\quad$ Median (25th-75th percentile) & $52(38-74)$ & $51(30-66)$ & \\
BUN, mg/dL & $32 \pm 18$ & $38 \pm 26$ & 0.346 \\
$\quad$ Median (25th-75th percentile) & $26(19-39)$ & $33(20-46)$ & 0.199 \\
Plasma albumin, g/dL & $3.9 \pm 0.6$ & $3.8 \pm 0.5$ & $<0.001$ \\
Plasma glucose, mg/dL & $120 \pm 39$ & $112 \pm 24$ & \\
NT-proBNP, pg/mL & $1,810(762-3,842)$ & $5,464(2,488-11,556)$ & \\
\hline
\end{tabular}

Means \pm SD or percent in the category unless indicated otherwise.

ure) codes. The mean overall follow-up was $11.5 \pm 6.4$ months to the date of HF-related death, 1st HF hospitalization, or last follow-up alive and without hospitalization. The study period was 1.5 years from the index BVA, and no patients were lost to follow-up. Renal function status was expressed as serum creatinine $(\mathrm{sCr})$ and $\mathrm{BUN}$ concentrations and estimated glomerular filtration rate (eGFR; in $\mathrm{mL} / \mathrm{min} / 1.73 \mathrm{~m}^{2}$ ) using the Modification of Diet in Renal Disease equation [11]. Echocardiographic measurements were obtained as recommended by the American Society of Echocardiography [12].

\section{Statistical Analyses}

Data are presented as means \pm SD or medians with 25 th and 75 th percentiles for continuous variables, and as numbers (\%) for categorical variables. One-way ANOVA was used for testing significant differences between PV groups. $p$ values and confidence intervals (CIs) are provided where appropriate. Survival was estimated using the Kaplan-Meier (K-M) log-rank analysis to test for differences in outcomes for a composite endpoint of HF-related mortality or 1st HF-related hospitalization after BVA. The composite endpoint was evaluated in univariate logistic regression and multivariable analyses using Cox proportional hazard models. Multivariable analysis first incorporated significant univariate predictors of outcome in the model which included common variables of HF-related risk. Those variables that did not retain statistical significance $(p>0.05)$ were removed from the final model. Results are presented as risk ratios (RR) with 95\% CIs and $p$ values. Statistical analyses were performed using SAS statistical software (version 9; SAS Institute, Cary, NC, USA) and JMP 8.

\section{Results}

For this analysis, BVA and clinical data were available on 110 patients who were prospectively followed for the composite outcome of HF mortality or 1st HF-related hospitalization after index BVA. Table 1 shows the clini$\mathrm{cal}$ and demographic features of this patient cohort at the time of BVA (baseline). This cohort was dichotomized based upon the measured median PV value of the cohort with PV $>+26 \%$ of the normal expected volume. Patients with PV above this median value were considered to have severe PV expansion $(n=55)$ and those below to have mild-moderate expansion $(n=55)$. Patients with severe $\mathrm{PV}$ expansion had higher $\mathrm{N}$-terminal pro-brain natriuretic peptide (NT-proBNP) levels and were more likely to have atrial fibrillation than patients with mild-moderate $\mathrm{PV}$ expansion, but otherwise the 2 subgroups were comparable (Table 2). Cohort median renal function parameters $\mathrm{sCr}(1.4 \mathrm{mg} / \mathrm{dL})$, eGFR $\left(51 \mathrm{~mL} / \mathrm{min} / 1.73 \mathrm{~m}^{2}\right)$, and BUN $(29 \mathrm{mg} / \mathrm{dL})$ were used to further partition the 2 subgroups for risk assessment. Table 3 shows specific intravascular volume profiles for the cohort based upon the category of PV expansion above and below the median. 
Table 3. Intravascular volume profiles stratified by the cohort's median (+26\%) plasma volume (PV)

\begin{tabular}{|c|c|c|c|}
\hline \multirow[t]{2}{*}{ Variable } & \multicolumn{2}{|c|}{ PV expansion } & \multirow{2}{*}{$\begin{array}{l}\text { Intergroup } \\
\text { comparison, } p\end{array}$} \\
\hline & $\begin{array}{l}<\text { median } \\
(n=(55)\end{array}$ & $\begin{array}{l}\geq \text { median } \\
(n=55)\end{array}$ & \\
\hline $\mathrm{PV}, \mathrm{L}$ & $3.6 \pm 0.8$ & $4.9 \pm 0.9$ & 0.001 \\
\hline Range & $2.3-6.3$ & $3.1-7.4$ & \\
\hline Excess/deficit, \% & $+10.6 \pm 9.7$ & $+51 \pm 19$ & $<0.001$ \\
\hline Range & -12 to +25 & +26 to +100.5 & \\
\hline RBCM, L & $2.4 \pm 0.5$ & $2.0 \pm 0.8$ & 0.002 \\
\hline Range & $1.1-3.7$ & $0.9-5.1$ & \\
\hline Excess/deficit, \% & $-7.0 \pm 25$ & $+12.8 \pm 22$ & $<0.001$ \\
\hline Range & -38 to +101 & -32 to +52 & \\
\hline $\mathrm{TBV}, \mathrm{L}$ & $5.6 \pm 1.4$ & $7.4 \pm 1.1$ & $<0.001$ \\
\hline Range & $3.2-9.1$ & $4.3-10.1$ & \\
\hline Excess/deficit, \% & $+4.1 \pm 13$ & $+36 \pm 13$ & $<0.001$ \\
\hline Range & -17 to +48 & +13 to +70.2 & \\
\hline
\end{tabular}

Means \pm SD (ranges) of the indicated parameter. TBV, total blood volume; RBCM, red blood cell mass defined as percent $(\geq-10$ to $\leq+10 \%)$ of normal expected volumes.

Per definition averaged PV in liters was significantly greater in patients with severe expansion. RBC mass was on average within the normal range or only mildly elevated (in the severe PV expansion group). Both patient subgroups, however, demonstrated marked heterogeneity in all volume profiles specifically with a range of $\mathrm{PV}$ in the severe expansion subgroup from +26 to $>+100 \%$ volume expansions.

Over the course of the 1.5-year study period, $41 / 110$ (37\%) patients experienced the composite endpoint of HF-related mortality or hospitalization (21/55 [38\%] in the severe PV expansion subgroup and 20/55 [36\%] in the mild-moderate PV expansion subgroup [RR 1.02, CI $0.55-1.89, p=0.959])$. However, when the 2 subgroups were dichotomized by the median cohort $\mathrm{sCr}$ concentration $(1.4 \mathrm{mg} / \mathrm{dL})$, there were differences in risk association based upon the extent of PV expansion. Figure 1a shows the K-M survival estimates for the composite endpoint in patients with severe PV expansion $(>+26 \%$ of normal PV) stratified by median sCr. An elevated $\mathrm{sCr}$ above the median in patients with severe PV expansion showed a substantially higher risk of poor outcome $(p=$ $0.02)$ compared with $\mathrm{sCr}<1.4 \mathrm{mg} / \mathrm{dL}$. Notably, in the setting of severe PV expansion, the K-M curves for the composite endpoint separated early in the course of followup: 30 vs. $5 \%$ risk at 4 months. This contrasts to a more uniform risk of approximately 24 vs. $20 \%$ risk, respectively, at 4 months for patients with mild-moderate PV expansion. This suggests that in the early time course fol- lowing index BVA despite severe PV expansion better renal function was associated with an outcome benefit. Importantly, there were no differences in clinical features or demographics between those with $\mathrm{sCr}$ above or below 1.4 $\mathrm{mg} / \mathrm{dL}$ in patients with severe PV expansion (data not shown). In contrast, in the subgroup with mild-moderate PV expansion (Fig. 1b), sCr above or below the cohort median was not associated with any change in risk ( $p=$ 0.578 ). Worse renal function was not associated with worse outcomes, and better renal function was not necessarily indicative of better outcomes.

Similar to the analysis with sCr, using median eGFR and BUN to risk stratify patients with severe and mildmoderate PV expansion, comparable relationships to the composite endpoint were demonstrated. eGFR below the median value for the cohort of $51 \mathrm{~mL} / \mathrm{min} / 1.73 \mathrm{~m}^{2}$ identified patients at increased risk in the subgroup with severe PV expansion (Fig. 2a) while the same cutoff did not stratify to an increase in risk in patients with mild-moderate PV expansion (Fig. 2b). BUN above the median value $(29 \mathrm{mg} / \mathrm{dL})$ identified increased risk in patients with severe PV expansion (Fig. 3a) while in patients with lesser PV expansion BUN above the median value was not associated with an increased risk compared to values below the median (Fig. 3b). Better renal function as reflected by higher eGFR and lower BUN appears in the setting of severe PV expansion to provide outcome benefit particularly as observed in the early time period (4-6 months) following index BVA. 


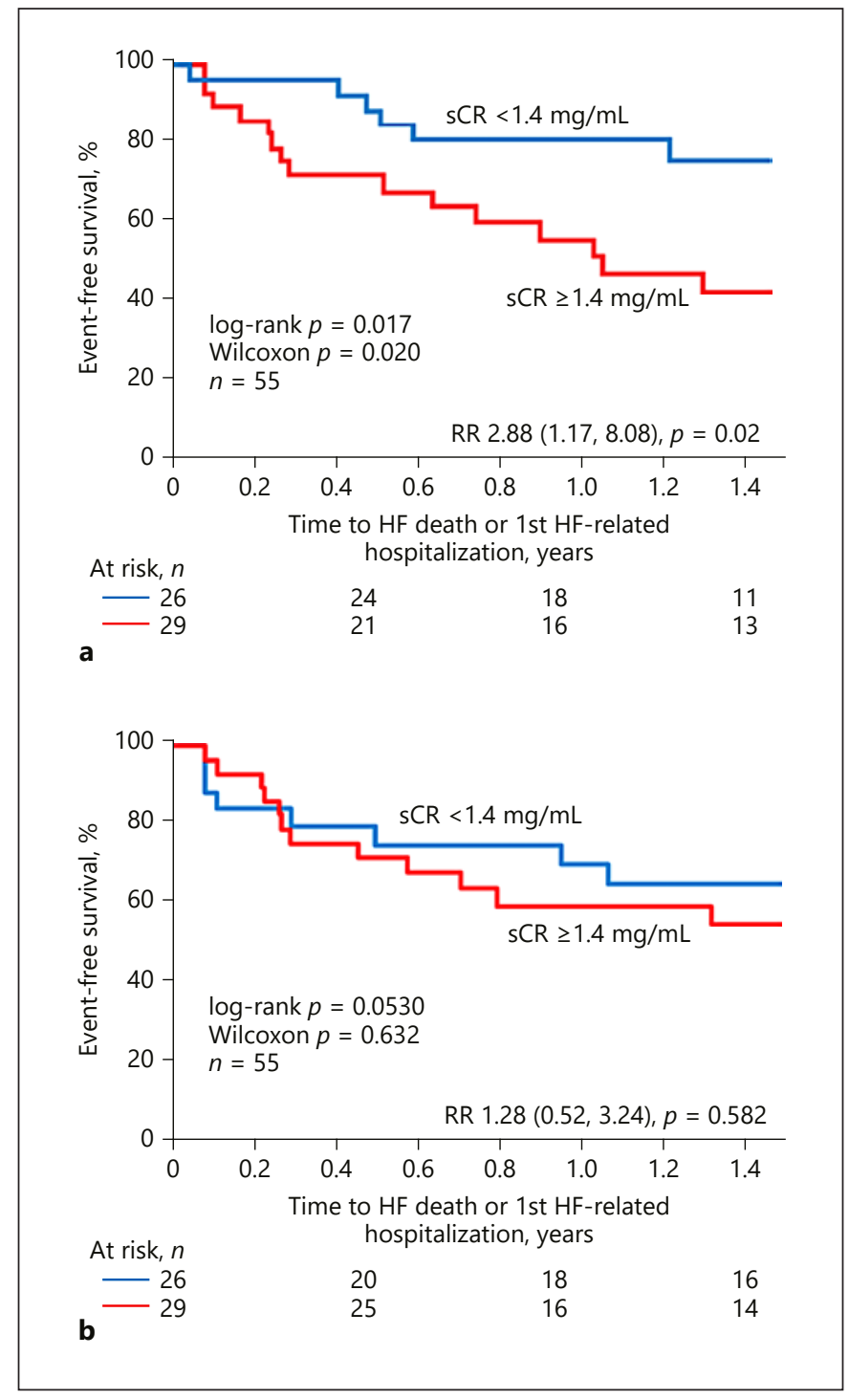

Fig. 1. a Risk stratification by cohort median serum creatinine $(\mathrm{sCr})$ in chronic heart failure (HF) patients with severe plasma volume (PV) expansion (median PV expansion $\geq+26 \%$ of the normal expected volume). b Risk stratification by cohort median serum creatinine in chronic HF patients with mild-moderate PV expansion (median PV expansion $<+26 \%$ of the normal expected volume).

Cox proportional hazard regression analysis was used to evaluate the association between common clinical parameters of increased risk and the composite endpoint of HF-related mortality or 1st HF-related hospitalization in relation to the severity of PV expansion. Variables tested univariately for predictive capacity were age, left-ventricular ejection fraction, NT-proBNP, presence of anemia $(\mathrm{Hb}<12 \mathrm{~g} / \mathrm{dL})$, diabetes, coronary artery disease, sleep
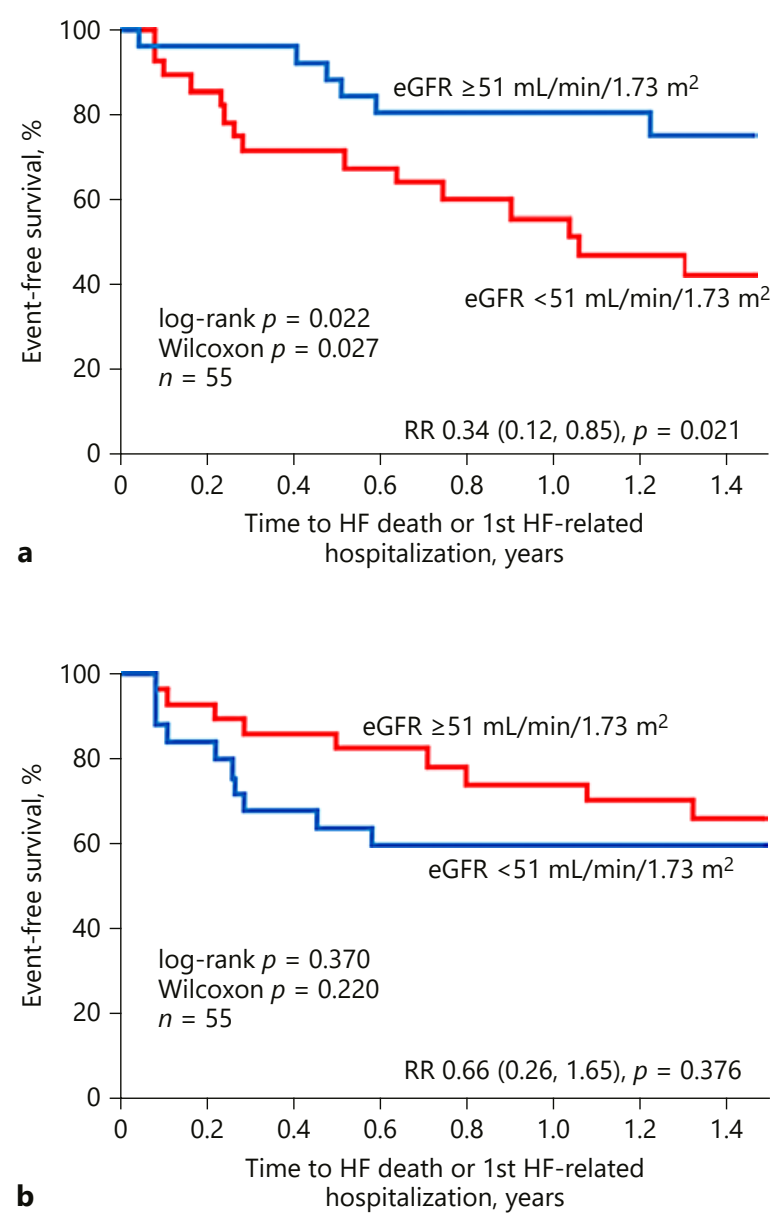

Fig. 2. a Risk stratification by cohort median eGFR in chronic heart failure (HF) patients with severe plasma volume (PV) expansion (median PV expansion $\geq+26 \%$ of the normal expected volume). b Risk stratification by cohort median eGFR in chronic HF patients with mild-moderate PV expansion (median PV expansion $<+26 \%$ of the normal expected volume).

apnea, and atrial fibrillation. In a subgroup of patients with PV expansion $\geq+26 \%$, none of these parameters was a univariate predictor of the composite outcome. In patients with mild-moderate PV expansion (PV <+26\%), anemia (RR 2.92 [CI 1.20-7.29], $p=0.018)$ and NT-proBNP (RR 2.80 [CI 1.10-7.36], $p=0.031$ ) were univariate predictors. Multivariable model analysis was undertaken to test for independent predictors of risk separately in the 


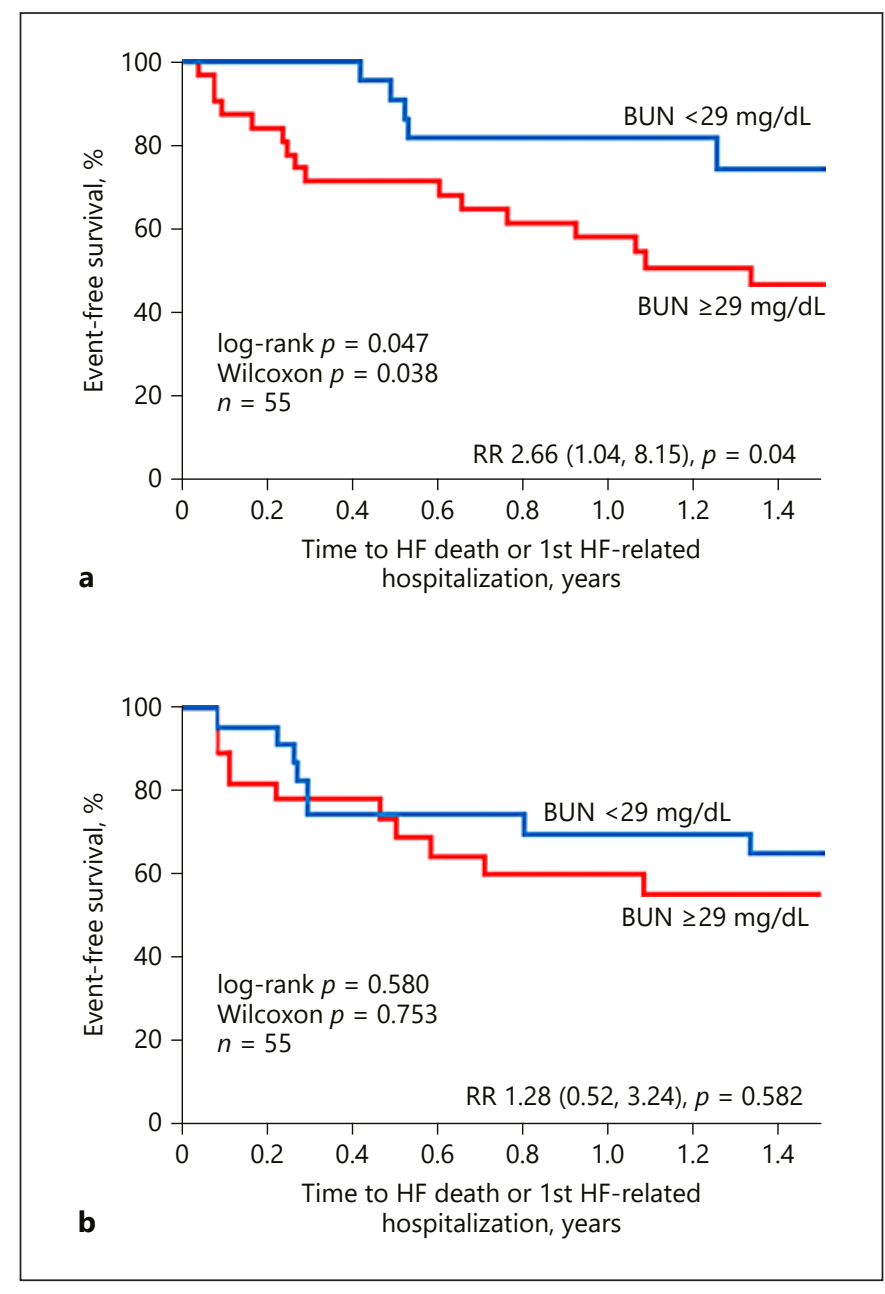

Fig. 3. a Risk stratification by cohort median BUN in chronic heart failure (HF) patients with severe plasma volume expansion (median PV expansion $\geq+26 \%$ of the normal expected volume). b Risk stratification by cohort median BUN in chronic HF patients with mild-moderate plasma volume (PV) expansion (median PV expansion $<+26 \%$ of the normal expected volume).

2 subgroups. For the subgroup with severe PV expansion when the markers of renal function (sCr, eGFR, BUN) were entered into the model, none of these univariate predictors persisted as independent predictor of outcome. Similarly, in the subgroup with mild-moderate PV expansion, neither the renal function biomarkers nor NTproBNP retained an independent predictive capacity. The presence of anemia in the subgroup of mild-moderate $\mathrm{PV}$ expansion, however, remained an independent predictor (RR 3.01 [CI 1.23-7.57], $p=0.016$ ) in the multivariable model. Using a Cox model testing for interaction of PV with renal function, a trend $(p=0.073)$ was

Volume-Kidney Interactions in Heart Failure demonstrated for the endpoint of death alone, but no interaction was demonstrated for the composite endpoint $(p=0.347)$.

Recognizing that the subgroup with PV $<+26 \%$ contained patients with very mild volume contraction $(\geq-$ $10 \%$ to $0[n=6])$ and very mild PV expansion ( $>0$ to $\leq+10 \%[n=20])$, we assessed differences relative to the group as a whole. No difference in sCr level was identified for the normal-range PV subgroup compared to the group as a whole (sCr $1.4 \pm 0.5$; median 1.4 (25th-75th CI 1.0-1.6). Median eGFR was also comparable to the overall subgroup at $52 \mathrm{~mL} / \mathrm{min} / 1.72 \mathrm{~m}^{2}$. Also, no difference in the composite outcome was demonstrated based upon stratified median sCr, BUN, or eGFR (log-rank $p=0.869)$. Similarly, patients with moderate PV expansion $>+10$ to $<+26 \%(n=29)$ demonstrated comparable sCr $(1.5 \pm 0.6$, median 1.4 [1.1-1.9 mg/dL]) and eGFR (median $51 \mathrm{~mL} /$ $\mathrm{min} / 1.73 \mathrm{~m}^{2}$ ). Composite outcomes were also not different when stratified by median $\mathrm{sCr}, \mathrm{BUN}$, or eGFR for this range of PV expansion (log-rank $p=0.417$ ). Overall, no separate parameter differences were identified for normal-range PV or mild-moderate PV expansion when compared with the subgroup as a whole.

\section{Discussion}

While progressive renal dysfunction has been repeatedly shown to be a strong predictor and probable modulator of outcome in patients with HF [1-4], the interaction of fluid volume overload with impaired renal function (CKD) has not been extensively studied $[13,14]$. The impact of intravascular volume and specifically the extent of subclinical PV expansion has not been analyzed, taken into account in risk assessment paradigms, or viewed as a modifiable risk factor in the management of HF patients with coexisting CKD. Here, we address the prevalence of quantitated subclinical intravascular volume overload and the interactions of the extent of PV expansion with different clinical biomarker parameters of CKD on HFrelated outcomes including mortality.

The principal findings of this analysis indicate that in patients with clinically stable, mild-moderately symptomatic HF who were considered "euvolemic" by clinical assessment, the presence of subclinical PV expansion is common ( $76 \%$ in this cohort), and, importantly, the extent of the intravascular volume excess from normalmild-moderate to severe PV expansion interacts with CKD severity to impact the predictive capacity of standard clinical biomarkers of renal function. Severe PV ex- 
pansion appears to underpin the capacity of clinical biomarkers of renal dysfunction in this cohort to identify patients at highest risk who may otherwise go undetected. From another perspective, it also appears that better renal function in the setting of severe PV expansion is somewhat protective or allows greater PV expansion to be better tolerated in patients with chronic HF.

We have shown in previous studies of patients with chronic HF that there is marked variability in and persistent congestion of intravascular volume when assessed quantitatively despite vigorous and uniform diuretic therapy [5]. In the current study of patients considered to be clinically "euvolemic" at the time of BVA assessment, higher sCr and BUN, and lower eGFR in the presence of severe PV expansion effectively identified patients at higher risk for HF-related mortality or hospitalization. Better kidney function (lower sCr and BUN, and higher eGFR) in the setting of severe PV expansion, however, appears to mitigate while worse kidney function exacerbates an already poor outcome. Thus, better kidney function appears to provide a compensatory advantage which allows HF patients to experience more significant subclinical volume overload with somewhat reduced risk and better clinical status.

In contrast, when PV is much less expanded (in this analysis lower than median expansion), the degree of $\mathrm{CKD}$ as reflected in the differences in clinical biomarkers is no longer a key identifier of risk or provides incremental information on risk assessment. From a kidney perspective in the setting of less PV expansion, the severity of kidney dysfunction appears to play less of a risk-stratifying role. This may in part relate to the kidney's marked sensitivity to fluid congestion where low renal medullary $\mathrm{pO}_{2}$ can be further impaired by severe volume expansion with renal tissue edema provoking greater renal hypoxia [15]. However, in the setting of lesser volume expansion and less congestion, oxygen delivery and medullary $\mathrm{pO}_{2}$ would be expected to be more favorable and similar in patients with either better or worse intrinsic renal function thus overriding at least in part the risk-stratifying capacity of CKD biomarkers. Another point is that while in the patients with severe PV expansion there was large variability in the extent of intravascular volume expansion among patients (Table 3 ), all had a severe degree of expansion. In contrast, the patients with less than severe PV expansion, who also demonstrated patient-to-patient variability in PV expansion, included normal and very mild, as well as, moderate PV expansion, and this spectrum of intravascular volume may have had a less uniform impact on outcome in relation to the $\mathrm{CKD}$ markers than in the severe PV expansion subgroup.
The findings of this analysis suggest that the extent of subclinical volume overload as well as functional status in the patients should be taken into account when interpreting the clinical significance of elevations in biomarkers of renal dysfunction in patients with coexisting chronic HF and kidney disease. Early in the follow-up period (4-6 months after BVA), the outcome risk was lower in patients with better renal function despite the presence of severe PV expansion compared to patients with comparable CKD but only mild-moderate PV expansion. This underscores the significance of volume-kidney interactions over time in ambulatory chronic HF patients and a possible mitigating effect on the risk of volume expansion. The observation, however, that the frequencies of the composite endpoint over 1.5 years were comparable in the 2 groups of PV expansion (36 and 38\%, respectively) suggests that factors in addition to renal dysfunction and their interactions contribute to outcomes.

The level of neurohormonal activation of the reninangiotensin-aldosterone system may have more of a role in contributing to outcome when there is less volume expansion and potentially inadequate intravascular filling. The finding that NT-proBNP was a univariate predictor in the mild-moderate PV expansion subgroup but blunted in the setting of severe PV expansion is suggestive that neurohormonal activation interacting with lesser degrees of PV expansion contributed to outcome risk while the parameters of renal dysfunction were less impactful. This is speculative and requires further study.

An additional factor is anemia, which has been shown to be a predictor of poor outcome in HF patients [16]. In this analysis, anemia (defined as venous $\mathrm{Hb}<12 \mathrm{~g} / \mathrm{dL}$ ) was demonstrated to be an independent risk factor in patients with mild-moderate PV expansion but not severe expansion. This likely reflects the impact of true anemia (defined as absolute reduction in RBC mass) on outcome in this subgroup compared with PV expansion-related dilutional reductions in $\mathrm{Hb}$ (pseudoanemia) in patients with severe PV expansion [17]. In this cohort, more patients in the mild-moderate PV expansion subgroup demonstrated true anemia $(29 / 55,53 \%)$ than in the severe expansion subgroup $(8 / 55,14.5 \%)$.

The extent of subclinical intravascular volume expansion appears to play a direct and interactive role in defining outcome risk in patients with chronic HF and coexisting CKD. Whether the interaction of volume status and CKD also has implications for response to standard HF therapies is an issue that requires further study and is not addressed in this analysis. The findings of this analysis support a conclusion that knowing quantitatively the ex-
56

Kidney Dis 2020;6:50-58 DOI: $10.1159 / 000502210$
Miller/Grill/Qian 
tent of intravascular expansion can significantly influence the interpretation of the potential impact of renal dysfunction on risk assessment and, therefore, the management approach in patients with ambulatory chronic $\mathrm{HF}$ and coexisting CKD.

\section{Study Limitations}

First, this is an observational single-center study of prospectively collected data from a tertiary referral medical center with potential limitations of selection bias and generalizability of findings. However, our practice serves the local population, and all patients were typical HF patients with NYHA class II stable chronic disease. Second, whether the findings of this study can be extrapolated to all classes/stages of HF patients, particularly more advanced $\mathrm{HF}$, with CKD cannot be concluded from these data and would require additional studies in appropriately defined HF-related patient cohorts. Third, while the biomarkers of renal function were univariate predictors of outcome, none remained independent in multivariable analysis. Colinearity of these parameters may have contributed to their being negated as independent predictors of increased risk when all were included in the analysis model. These parameters, however, would in clinical practice likely be evaluated as individual indicators of risk assessment and, therefore, preserve their value in this analysis. Fourth, the lack of serial PV and biomarker measurements over the time course of the study limit the ability to account for changes in PV status (crossover) and renal function, which may have occurred and possibly influenced risk assessment and outcomes over the extended period of follow-up.

\section{Conclusions}

In patients with stable clinically assessed "euvolemic" chronic HF and comorbid CKD, subclinical PV expansion is common, and, importantly, the extent of PV expansion interacts with CKD severity to significantly impact the capacity of standard clinical biomarkers of renal function to predict outcomes of HF-related mortality and hospitalization. Lesser degrees of PV expansion appear to reduce the outcome impact of these biomarkers, whereas with greater PV expansion the same biomarkers effectively stratify patients at higher risk. Therefore, the quantitative assessment of intravascular volume can help inform assessment of risk and, therefore, may importantly impact the direction for a more individualized approach to management. Further study of volume-kidney interactions is warranted to better understand underlying mechanisms and their impact in patients with varying degrees of $\mathrm{HF}$ and co-existing CKD.

\section{Statement of Ethics}

This study was approved by the Mayo Foundation Institutional Research Review Board as required by Minnesota Statute 144.335/CFR 21 (Part 50). The project was approved by the Mayo Clinic Institutional Review Board. All participants consented to participate in the study.

\section{Disclosure Statement}

The authors have no conflict of interest to declare.

\section{References}

1 Löffler AI, Cappola TP, Fang J, Hetzel SJ, Kadlec A, Astor B, et al. Effect of renal function on prognosis in chronic heart failure. Am J Cardiol. 2015 Jan;115(1):62-8.

2 Damman K, Valente MA, Voors AA, O'Connor CM, van Veldhuisen DJ, Hillege HL. Renal impairment, worsening renal function, and outcome in patients with heart failure: an updated meta-analysis. Eur Heart J. 2014 Feb;35(7):455-69.

3 van Veldhuisen DJ, Ruilope LM, Maisel AS, Damman K. Biomarkers of renal injury and function: diagnostic, prognostic and therapeutic implications in heart failure. Eur Heart J. 2016 Sep;37(33):2577-85.
4 Metra M, Davison B, Bettari L, Sun H, Edwards $\mathrm{C}$, Lazzarini $\mathrm{V}$, et al. Is worsening renal function an ominous prognostic sign in patients with acute heart failure? The role of congestion and its interaction with renal function. Circ Heart Fail. 2012 Jan;5(1):5462.

5 Miller WL, Mullan BP. Understanding the heterogeneity in volume overload and fluid distribution in decompensated heart failure is key to optimal volume management: role for blood volume quantitation. JACC Heart Fail. 2014 Jun;2(3):298-305.

6 Miller WL. Fluid volume overload and congestion in heart failure: Time to reconsider pathophysiology and how volume is assessed. Circ Heart Fail. 2016 Aug;9(8):e002922.
7 Feldschuh J. Blood volume measurements in hypertensive disease. In: Larah JH, Brenner BM, eds. Hypertension: pathology, diagnosis, and management. New York, NY: Raven Press; 1990.

8 Feldschuh J, Enson Y. Prediction of the normal blood volume. Relation of blood volume to body habitus. Circulation. 1977 Oct;56(4 Pt 1):605-12.

9 Dworkin HJ, Premo M, Dees S. Comparison of red cell and whole blood volume as performed using both chromium-51-tagged red cells and iodine-125-tagged albumin and using I-131-tagged albumin and extrapolated red cell volume. Am J Med Sci. 2007 Jul; 334(1):37-40. 
10 Fairbanks VF, Klee GG, Wiseman GA, Hoyer JD, Tefferi A, Petitt RM, et al. Measurement of blood volume and red cell mass: re-examination of 51Cr and 125I methods. Blood Cells Mol Dis. 1996;22(2):169-86.

11 Levey AS, Coresh J, Balk E, Kausz AT, Levin A, Steffes MW, et al.; National Kidney Foundation. National Kidney Foundation practice guidelines for chronic kidney disease: evaluation, classification, and stratification. Ann Intern Med. 2003 Jul;139(2):137-47.

12 Lang RM, Badano LP, Mor-Avi V, Afilalo J, Armstrong A, Ernande L, et al. Recommendations for cardiac chamber quantification by echocardiography in adults: an update from the American Society of Echocardiography and the European Association of Cardiovascular Imaging. J Am Soc Echocardiogr. 2015 Jan;28(1):1-39.e14.

13 Nijst P, Martens P, Dupont M, Tang WH, Mullens W. Intrarenal flow alterations during transition from euvolemia to intravascular volume expansion in heart failure patients. JACC Heart Fail. 2017 Sep;5(9):672-81.

14 Westenbrink BD, Visser FW, Voors AA, Smilde TD, Lipsic E, Navis G, et al. Anaemia in chronic heart failure is not only related to impaired renal perfusion and blunted eryth- ropoietin production, but to fluid retention as well. Eur Heart J. 2007 Jan;28(2):166-71.

15 Ding X, Cheng Z, Qian Q. Intravenous fluids and acute kidney injury. Blood Purif. 2017; 43(1-3):163-72.

16 Felker GM, Adams KF Jr, Gattis WA O'Connor CM. Anemia as a risk factor and therapeutic target in heart failure. J Am Coll Cardiol. 2004 Sep;44(5):959-66.

17 Miller WL, Mullan BP. Peripheral venous hemoglobin and red blood cell mass mismatch in volume overload systolic heart failure: implications for patient management. J Cardiovasc Transl Res. 2015 Oct;8(7):404-10. 